Introduction Measles is a preventable disease through vaccination. In spite of the vaccine introduced in $1979^{[1]}$, an important outbreak is ongoing in Romania since $2017^{[2]}$.

We performed a retrospective study, which included 330 patients with measles, aiming to obtain data for vaccination status and possible correlations with clinical complications. The identified viral genotype was B3, strain MVs/Dublin.IRL/8.16, which is not the usual one circulating in Romania [1], [2].

Methods Retrospective statistical data analysis was performed (SPSS), based on information obtained from medical documents (clinical forms, complications, immunization status).

Inclusion criteria - patients presenting with clinical symptoms (fever; cough; coryza; maculopapular exanthema) admitted into 'Dr V. Babes' Clinical Hospital, in a 2 year period (2016-2017). Cases were stratified (severe/mild) based on the presence of complication (pneumonia, diarrhoea, dehydration). Cases without laboratory confirmation, using the ELISA method for specific serum IgM antibodies or viral RNA detection by RT-PCR, were excluded from the study.

Results The most affected age categories were between 1-4 years with $40.9 \%(\mathrm{~N}=135)$, similar to reported ECDC data ${ }^{[1]}$.

In the studied group, $50.9 \%(\mathrm{~N}=168)$ of the patients had not been vaccinated, while $14.2 \%(\mathrm{~N}=47)$ were infected in spite of being immunized with one or two MMR vaccine doses. For 115 patients $(34.8 \%)$ the immunization status was unknown.

The non-immunised patients were more likely to develop a severe form of the disease $(92.9 \%, \mathrm{~N}=156)$, in comparison with the vaccinated individuals who associated a complication in $85.1 \%(\mathrm{~N}=40)$ of cases.

A mild form of Measles was reported in 7.1\% (N=12) of unvaccinated patients and in $14.9 \% \quad(\mathrm{~N}=7)$ of previously immunised individuals.

It was a statistically significant correlation between the vaccination status and the clinical form of the disease $\left(\chi^{2}-\right.$ 334.203, df-4, $\mathrm{P}<0.001)$.

Discussions In Romania, the vaccination coverage for the first dose of MMR was in $2017-86 \%$ and for the second dose $75 \%$ [4].

In order to respond to the outbreak, Romania has lowered the age of administering the first vaccine dose from 12 to 9 months $_{[5]}$.

Since 2017 the number of reported cases has increased with $53.6 \%(\mathrm{~N}=15.971)$, and to date, 60 deaths have been reported, the majority of which occurred in persons who were not vaccinated [6], [7].

Conclusions Drop in vaccination rate is the main reason for the measles outbreak in Romania.

Vaccination statistically represented a protection factor against complications.

Better public information may improve the general acceptance of the vaccination scheme.

\section{GP191 DYSREGULATION OF THE INFLAMMASOME IN CHILDREN WITH DOWN SYNDROME}

\footnotetext{
${ }^{1}$ Amy P Worrall*, ${ }^{1}$ Eleanor Gallagher, ${ }^{2,3}$ Dean Huggard, ${ }^{3}$ Lynne Kelly, ${ }^{3,2,4,5}$ Eleanor Molloy. ${ }^{1}$ Trinity College Dublin, Dublin, Ireland; ${ }^{2}$ Department of Paediatrics, Tallaght University Hospital NCH/TUH, Dublin, Ireland; ${ }^{3}$ Trinity Translational Medicine Institute, Trinity College Dublin, Dublin, Ireland; ${ }^{4}$ Our Lady's Children's Hospital Crumlin, Dublin, Ireland; ${ }^{5}$ The Coombe Women and Infant's University Hospital, Dublin, Ireland
}

10.1136/archdischild-2019-epa.252
Aims Children with Down Syndrome (DS) are at an increased risk of infection and sepsis, and other inflammatory conditions such as arthritis. Various forms of immune dysregulation have been documented in DS including lymphopaenia, altered serum cytokines, reduced response to vaccinations as well as leukaemias. The NLRP3 (NLR family pyrin domain containing 3) inflammasome is a multiprotein complex that generates pro-inflammatory cytokines as part of the innate immune response to infection andis implicated in several inflammatory disorders. NLRP3 is also an important target for anti-inflammatory therapies. We aimed to characterize gene expression of the NLRP3 inflammasome in children with DS.

Methods Peripheral venous whole blood samples were collected from children with DS $(n=18)$ and healthy age-matched paediatric controls $(n=10)$. Whole blood samples were treated with lipopolysaccharide (LPS), melatonin or both. Whole blood RNA was isolated, cDNA was synthesized and analysed by quantitative PCR for expression of NLRP3, ASC (Apoptosis-associated speck like protein containing a caspase recruitment domain) and IL-1 $\beta$ (Interleukin-1- $\beta$ ). Statistical analysis was performed using ANOVA and t-test with Graphpad Prism Version 7.0.

Results The expression of NLRP3 and IL-1 $\beta$ was similar between children with DS and controls at baseline. ASC was significantly reduced in children with DS $(p=0.0003)$. Both cohorts demonstrated a significant rise in the expression of IL-1 $\beta$ following treatment with LPS $(p \leq 0.0001)$. Melatonin significantly attenuated IL- $1 \beta$ expression in children with DS, this was not demonstrated for the control group $(p \leq 0.0001)$.

Conclusion Dysfunction of various elements of the inflammasome and its associated regulatory genes could be responsible for increased susceptibility to infection in children with DS. Melatonin significantly reduces IL-1 $\beta$ expression following LPS and may be beneficial as an adjunctive therapy in children with DS.

\section{GP192 THE TREATMENTS AND PROGNOSIS OF 50 CASES OF CGD PATIENTS IN SINGLE CENTER OF CHINA}

JIANG Liping*, Yao CAO, XIANG Qing, WANG Shiyu, XIAO Min. Children's Hospital of Chongqing Medical University, Chongqing, China

\subsection{6/archdischild-2019-epa.253}

Background and aims Chronic granulomatous disease (CGD) is a rare primary immunodeficiency caused by mutations in the $\mathrm{NADPH}$ oxidase in phagocytic leucocytes, leading to severe life-threatening bacterial and fungal infections. The most common treatments are prophylactic antibiotics sulfamethoxazole (SMZ) and occasionally antifungal or anti-tuberculosis therapy. Haemopoietic stem-cell transplantation (HSCT) is currently the only curative option for these patients. We summarized the treatments and prognosis of 50 cases of CGD patients in a single center in Chongqing, China.

Methods 50 CGD patients were recruited between 2005 and 2017 based on clinical diagnosis, survival analysis was performed. The primary endpoints were overall survival and event-free survival (EFS), frequency of infections, incidence of acute and chronic graft-versus-host disease (GVHD) after at least 18 months of follow-up.

Results SMZ was begun for all patients since diagnosis. The overall survival was $80 \%$. Among the 50 patients enrolled, 20 X-linked CGD patients received HSCT with a success rate of 95\%. 10 HLA-matched related-donor and 10 HLA-matched 
unrelated-donor transplants were done. The cumulative incidence of acute GVHD of grade I-II and grade III-IV were $60 \%$ and $5 \%$, respectively. The myeloid donor chimerisms were all at least 94\% except one $86.1 \%$. Conservative treatment and HSCT decreased separately the frequency of infections to $5.1 \%$ and nearly 0 per 100 patient-months in patients.

Conclusions Severe infections were the main cause of death and the overall mortality was still high in China. HSTC is a potentially curative therapy for CGD leading resolution of infections and complications. CGD children who had undergone HSCT have better quality of life and fewer infections compared with those treated conservatively.

\section{GP194 PERIODIC FEVER-THE IRISH PFAPA STORY SO FAR}

${ }^{1}$ Ana Louise Hawke*, 'Timothy Ronan Leahy, ${ }^{1}$ Jayne M MacMahon, ${ }^{1} E m m a$ Jane MacDermott, ${ }^{2}$ Karina Butler, 'Patrick Gavin, 'Orla G Killeen. 'Our Lady's Children's Hospital, Crumlin, Dublin, Ireland; 'Our Lady's Children's Hospital, Crumin, Dublin, Ireland

\subsection{6/archdischild-2019-epa.254}

Introduction Periodic fever, aphthous stomatitis, pharyngitis and cervical adenitis (PFAPA) syndrome is the most common autoinflammatory disorder in childhood, with multifactorial, polygenic causes postulated.

Objective To appraise the clinical features, inflammatory characteristics and management of children with PFAPA attending a tertiary Autoinflammatory Clinic.

Methods A retrospective observational chart review of all children with confirmed clinical or suspected PFAPA attending the autoinflammatory clinic at Our Lady's Children's Hospital, Dublin from January 2016.

Data were collected on basic demographics, route of referral, symptoms and signs and inflammatory markers during disease episodes (febrile) and non-episodes. Molecular gene analysis if performed, were included. Documentation of all therapeutic agents to date was collated.

Results Thirteen children were identified as having PFAPA.

The median age of disease onset was 16 months, (4 months to 4 years). The route of referral was via Immunology (4 patients), Rheumatology (6 patients) and Infectious disease (3 patients).

All children presented with episodic, recurrent febrile episodes with associated features. (table 1). Median range of duration of episodes was 3-4 days.

\begin{tabular}{|c|c|c|c|c|c|}
\hline \multirow[t]{7}{*}{ Associated Symptoms: } & \multicolumn{2}{|l|}{ Stomatitis } & \multicolumn{3}{|l|}{8} \\
\hline & \multicolumn{2}{|c|}{ Tonsillitis/Pharyngitis } & 8 & & \\
\hline & \multicolumn{2}{|c|}{ Cervical Adenitis } & 7 & & \\
\hline & \multicolumn{2}{|c|}{ Lethargy } & 6 & & \\
\hline & \multicolumn{2}{|l|}{ Rash } & 4 & & \\
\hline & \multicolumn{2}{|l|}{ Anorexia } & 4 & & \\
\hline & \multicolumn{2}{|l|}{ GI upset } & 12 & & \\
\hline Periodicity of disease & $<2$ weeks & 1 patient & Flare & $2-3$ days & 5 patients \\
\hline flare & 2-4 weeks & 10 patients & Duration & $4-5$ days & 7 patients \\
\hline & 4-6 weeks & 2 patients & & $6-7$ days & 1 patient \\
\hline
\end{tabular}

$69 \%$ of patients had documented raised inflammatory markers during a flare, with $84 \%$ having high serum amyloid
A (SAA) levels, the highest documented being $1220 \mathrm{mg} / \mathrm{l}$ (< 10 normal). Genetic testing in 6 children was negative for other causes of hereditary autoinflammatory disorders.

11 patients had a significant response to an initial trial of corticosteroids, 2 reported rebound flares. Colchicine was the treatment of choice (11), 9 who had a good response. Tonsillectomy was performed in 5 patients, 3 of whom reported benefit. Biologic agents, Anakinra (2) and Adalimumab (1) were instituted in those refractory to colchicine with variable response.

Conclusion This study gives an over view of the burden of disease imposed by PFAPA on an Irish population. All the children presented with fevers, not all had the triad of aphthous stomatitis, pharyngitis and cervical adenitis.

The majority of patients had relief of symptoms with an initiation trial of corticosteroid. Colchicine was the most frequently used therapeutic agent to prevent disease flares.

Tonsillectomy and biological agents are potential alternative options in some resistant/severe cases.

\section{GP195 ANTIBIOTIC THERAPY IN CHILDREN HOSPITALIZED DUE TO RSV LOWER RESPIRATORY TRACT INFECTION}

1,2Małgorzata Czajkowska* 1,2 August Wrotek, 1,2Teresa Jackowska. 'Department of Pediatrics, the Medical Centre of Postgraduate Education, Marymoncka 99/103, 01-813,

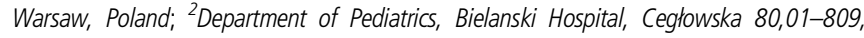
Warsaw, Poland

\subsection{6/archdischild-2019-epa.255}

Background Polish guidelines do not support antibiotic therapy in children with RSV (Respiratory Syncytial Virus) infection with few exceptions. We analyzed the frequency and reasons for antibiotic treatment in children under 2 years of age to assess the most important drivers in antibiotic use, and its correlation with routinely used inflammatory markers.

Material and Methods 198 children (median age3 months) were hospitalized due to RSV lower respiratory tract infection (RSV-LRTI) in two consecutive seasons (2016/2017 and 201718). RSV infection was confirmed with rapid antigen test and/or polymerase chain reaction. 197 children were enrolled into the study.

Results Antibiotics were used in 39\% (76/197) patients, including 6 patients $(8 \%)$ who received broad-spectrum antibiotics. The main reason for antibiotic use was pneumonia $(57 \% ; 43 /$ 76), followed by acute otitis media (AOM) in 18\% (14/76), lack of improvement $(15 \% ; 11 / 76)$, and urinary tract infections (UTI) in $10 \%(8 / 76)$. Children who received antibiotics were older (median 3.5 vs. 2 months; p<0.01), presented with statistically higher C-Reactive Protein $(6.65$ vs. $2.1 \mathrm{mg} / \mathrm{L}$; $\mathrm{p}<0.01)$, andprocalcitonin (PCT) level $(0.13$ vs. $0.09 \mathrm{ng} / \mathrm{mL}$; $\mathrm{p}<0.01)$, white blood cells $\left(12\right.$ vs. $10.6 \times 10^{\wedge} 3$ cell $/ \mathrm{uL}$; $\mathrm{p}=\mathbf{0 . 0 4})$ and absolute neutrophil count (4.09 versus $2.17 \times 10^{\wedge} 3$ cell $\left./ \mathrm{uL} ; \mathbf{p}<0.01\right)$. The differences had no clinical significance. Patients were further analyzed in the subgroups: pneumonia, AOM, lack of improvement and UTI group, but no clinically relevant differences were found. Only patients who were given broad-spectrum antibiotics had clinically and statistically significance in PCT (1.95 vs. $0.1 \mathrm{ng} / \mathrm{mL} ; \mathrm{p}=0.01)$. AOM in older children were at 3.96-fold increased risk of antibiotic therapy (95\%CI: 1.1-14.7). Patients who received antibiotics required longer hospital stay (11.5 vs. 9 days, $\mathrm{p}<\mathbf{0 . 0 1}$ ), especially when broad-spectrum antibiotics were given (17 versus 9 days, $\mathrm{p}<0.01$ ). 